\title{
Burnout in healthcare workers managing chronic patients with disorders of consciousness
}

\author{
O. GOSSERIES ${ }^{1,2}$, A. DEMERTZI ${ }^{1,2}$, D. LEDOUX ${ }^{1,3}$, M.-A. BRUNO ${ }^{1,2}$, \\ A. VANHAUDENHUYSE ${ }^{1,2}$, A. THIBAUT ${ }^{1,2}$, S. LAUREYS ${ }^{1,2}, \&$ C. SCHNAKERS ${ }^{1,2}$ \\ ${ }^{1}$ Coma Science Group, Cyclotron Research Centre, University of Liège, Liège, Belgium, ${ }^{2}$ Department of Neurology, and \\ ${ }^{3}$ Department of Intensive Care, University Hospital of Liège, Liège, Belgium
}

(Received 11 December 2011; revised 22 April 2012; accepted 14 May 2012)

\begin{abstract}
Objectives: The aim of this study was to assess the presence of burnout among professional caregivers managing patients with severe brain injury recovering from coma and working in neurorehabilitation centres or nursing homes.

Methods: The Maslach Burnout Inventory was sent to 40 centres involved in the Belgian federal network for the care of vegetative and minimally conscious patients. The following demographic data were also collected: age, gender, profession, expertise in the field, amount of time spent with patients and working place.

Results: Out of 1068 questionnaires sent, 568 were collected ( $53 \%$ response rate). Forty-five were excluded due to missing data. From the 523 healthcare workers, $18 \%(n=93)$ presented a burnout, $33 \%(n=171)$ showed emotional exhaustion and $36 \%(n=186)$ had a depersonalization. Profession (i.e. nurse/nursing assistants), working place (i.e. nursing home) and the amount of time spent with patients were associated with burnout. The logistic regression showed that profession was nevertheless the strongest variable linked to burnout.

Conclusions: According to this study, a significant percentage of professional caregivers and particularly nurses taking care of patients in a vegetative state and in a minimally conscious state suffered from burnout. Prevention of burnout symptoms among caregivers is crucial and is expected to promote more efficient medical care of these challenging patients.
\end{abstract}

Keywords: Emotional exhaustion, depersonalization, vegetative state, minimally conscious state

\section{Introduction}

Working with patients in a chronic vegetative or minimally conscious state is a real clinical challenge. Vegetative state, newly called 'unresponsive wakefulness syndrome', is characterized by the presence of arousal in absence of awareness showing only reflexive motor responses $[1,2]$, whereas the minimally conscious state has been defined by the presence of inconsistent but reproducible goaldirected behaviours [3]. Some of these patients remain for many years in those states without being able to communicate with the outside world. Hence, their medical management might have a significant psychological impact on healthcare teams. Indeed, the importance of motor (e.g. paralysis and severe spasticity), physiological (e.g. tracheotomy and gastrostomy) and cognitive (e.g. memory disorders, aphasia or lack of adequate communication) deficits involves intensive management. In light of patients full dependence on the medical team for all daily activities, the practical and emotional challenges caregivers are faced with are evident. The emotional aspect is of particular importance when one considers the low chance of recovery in this patient's population, the risk of life-threatening medical complications, the possible premature death of patients and potential conflicts with families. 
All these components can contribute to serious psychological distress and more particularly to burnout [4].

Burnout syndrome can be defined by physical and psychological emotional exhaustion, depersonalization and a low sense of personal accomplishment [4]. This syndrome occurs in response to both physical and psychological stress encountered in the workplace and is relatively common in professionals taking care of patients with critical illness, particularly in medical areas such as psychiatry, geriatric, oncology, emergency, surgery and intensive care units [5-9]. Burnout may decrease quality-of-life of healthcare professionals, generating symptoms such as anxiety, irritability, mood swings, insomnia or sense of failure. It has also been associated with substance abuse, early retirement, physical illness, absenteeism, depression and even death [10-12]. Caregivers work in emotionally-charged situations that may lead to conflicts with patients, their families and other members of the medical staff [13]. All those factors result in decreased quality-of-care and patients' safety $[14,15]$.

Some studies suggest that stress observed in nursing staff working in rehabilitation services may be related to the diagnosis of the patients and the severity of the lesions [16, 17]. Another study including 16 caregivers working with patients in a vegetative state showed that the level of anxiety and depression was correlated with unsatisfactory relationships with family and possible death of patients [18]. However, specifically the presence of burnout among medical teams managing patients diagnosed in a vegetative state and in a minimally conscious state has never been investigated. Therefore, it was decided to assess the burnout in healthcare workers managing patients with severe brain injury and impaired consciousness.

\section{Methods}

Forty institutions (neurorehabilitation centres and nursing homes) involved in the Belgian federal project on the management of patients with impaired consciousness were contacted in order to obtain their consent to participate in the study. Following agreement, the Maslach Burnout Inventory (MBI; French or Dutch version) [4], a standardized questionnaire assessing burnout syndrome, was sent by regular mail together with a form explaining the aim of the study. Participants were asked to fill in the questionnaire only if they wanted to participate in the study. The questionnaire was anonymously completed and was returned by the referent of each institution. This procedure was approved by the ethics committee of the Faculty of Medicine of the University of Liège in Belgium.

The MBI questionnaire, which has become the 'almost universally accepted gold standard to assess burnout' [19] due to its high reliability and validity $[4,20]$, consists of 22 personal statements in which the participant has to estimate the frequency at the professional level on a 7-point Likert scale, ranging from never experiencing those feelings ( 0 points) to having those feelings every day ( 7 points). The total score varies from $0-132$. The questionnaire measures three components of burnout: emotional exhaustion, depersonalization and personal accomplishment. The emotional exhaustion sub-scale contains nine items such as 'I feel emotionally drained by my work' with a score varying from 0-54. The depersonalization sub-scale includes five items such as 'I do not really care what happens to my patients', with a score from $0-30$. The personal accomplishment sub-scale is composed of eight items such as 'I have accomplished many worthwhile things in this job' with a score ranging from 0-48. Each of the three components was divided into low, moderate and high level according to the recommendation of the authors of the MBI. High mean scores on emotional exhaustion and depersonalization scales and low mean scores on personal accomplishment are suggestive of burnout [21]. However, for the determination of the level of burnout (i.e. moderate or high), the methodology used in previous studies $[5,12]$ was adopted. Since the personal accomplishment does not always correlate negatively with the first two sub-scales, it was removed specifically from the calculation of burnout. Burnout was therefore considered moderate if the participant had a score $>26$ at the emotional exhaustion scale or a score $>9$ at the depersonalization scale and a high burnout was considered if the participant had both a score $>26$ at the emotional exhaustion scale and a score $>9$ at the deperzonalization scale $[4,5,12]$.

The following demographic data were also collected: age, gender, profession (i.e. physician, nurse/ nursing assistant, physio/speech/occupational therapist or psychologist/social worker), expertise in the care of patients with disorders of consciousness (i.e. $<4$ years, between $4-9$ or $>9$ years), amount of time spent with these patients (i.e. hours per week) and working place (i.e. neurorehabilitation centre or nursing home).

Statistical analyses were performed using STATA software (Stata Statistical Software; Release 11. College Station, TX: StataCorp LP 2009). A series of Chi-square tests were conducted to determine if the proportion of participants was higher depending on the presence or absence of burnout for the following variables: gender, profession, expertise and 
Table I. Participants' demographics $(n=523)$ according to working place, profession and expertise.

\begin{tabular}{llr}
\hline Variables & \multicolumn{1}{c}{ Sub-category } & Participants \\
\hline Working place & neurorehabilitation centre & $44 \%$ \\
& nursing home & $56 \%$ \\
Profession & physician & $5 \%$ \\
& nurse & $47 \%$ \\
& nursing assistant & $21 \%$ \\
& physiotherapist & $11 \%$ \\
& speech therapist & $2 \%$ \\
& occupational therapist & $8 \%$ \\
Expertise & psychologist & $3 \%$ \\
& social worker & $3 \%$ \\
& $<4$ years & $44 \%$ \\
& $4-9$ years & $40 \%$ \\
& $>9$ years & $16 \%$ \\
\hline
\end{tabular}

working place. Kruskal-Wallis tests were also performed to determine if the age of the caregiver and the time spent with patients were different depending on the presence or absence of burnout. Same analysis using Chi-square and Kruskal-Wallis tests were replicated for the three sub-components of burnout (i.e. emotional exhaustion, depersonalization and personal accomplishment). Finally, a multivariate analysis with a logistic regression (stepwise backward selection) was performed to highlight the variables associated with burnout. Results were considered significant at $p<0.05$.

\section{Results}

All centres included in the federal project participated in the study. From the 1068 questionnaires sent out, 568 were collected ( $53 \%$ response rate). Forty-five questionnaires were removed from the analysis due to too many missing responses or unclear answers. Five hundred and twenty-three questionnaires were therefore included in the analysis. The average age of participants was $37(S D=10)$ years (449 women) and the average time spent with patients who have suffered severe brain damage was $19(\mathrm{SD}=14)$ hours per week. Table I shows the percentage of participants for the working place, profession and expertise.

According to the MBI questionnaire, 93 participants presented a burnout (18\%). A moderate burnout was observed in 76 responders (15\%) and 17 caregivers (3\%) showed a high burnout (Figure 1). Moderate-to-high emotional exhaustion was present in 171 participants (33\%) and moderate-to-high depersonalization in 186 responders $(36 \%)$. Personal accomplishment was considered low in 15 participants (3\%) (Table II). Among the burnout responders, 63 out of $93(68 \%)$ presented a high emotional exhaustion, $47(50 \%)$ had a high depersonalization and $10(11 \%)$ showed a low personal accomplishment.

Globally, a difference between proportions of participants with and without burnout was found for the profession (Table III). Burnout was higher among nurses (24\%) and nursing assistants (23\%) than other occupations $(8.5 \%)$. The time spent with patients was also a factor of burnout with burnout participants spending more time with patients $(23 \pm 14$ hours $)$ than participants who did not present the syndrome (18 \pm 14 hours). Finally, a difference was also observed for the working place with more burnout in nursing homes $(23 \%)$ than in rehabilitation centres (14\%). No significant result was obtained for the age, gender and expertise (Table IV).

For the emotional exhaustion sub-component, only a difference for the profession was observed with nurses and nursing assistants presenting more exhaustion than other jobs. No significant result was obtained for the other variables (i.e. working place, age, gender, expertise and time) (Table IV).

For the depersonalization sub-component, no significant difference between proportions for the profession was obtained. However, the proportion of participants with high depersonalization was greater among nurses and nursing assistants $(12 \%)$ as compared to other professions (4\%). A difference according to the time was also measured with a greater number of hours spent with patients for participants with high depersonalization $(27 \pm 13$ hours) than for participants with a low $(18 \pm 14$ hours) to moderate ( $19 \pm 13$ hours) depersonalization. No significant result was obtained for the working place, age, gender and expertise (Table IV).

For the personal accomplishment sub-component, no significant difference was obtained for the profession. The proportion of responders with a low achievement seemed nevertheless greater among nurses and nursing assistants (3\%) than for other professions $(0.2 \%)$. A difference in the age was also found with a lower age for participants with higher personal accomplishment $(37 \pm 10)$ than for participants? with a low-to-moderate personal accomplishment $(40 \pm 9)$. A difference was also obtained depending on the time spent with patients, the number of hours was numerically less for participants with high personal accomplishment $(18 \pm 13$ hours) than for participants with low ( $36 \pm 7$ hours) or moderate ( $22 \pm 15$ hours) personal accomplishment. No significant result was found for the working place, gender and expertise. Table IV shows the chi-square values and $p$-values for the burnout and its three sub-components (emotional exhaustion, depersonalization and personal accomplishment). 


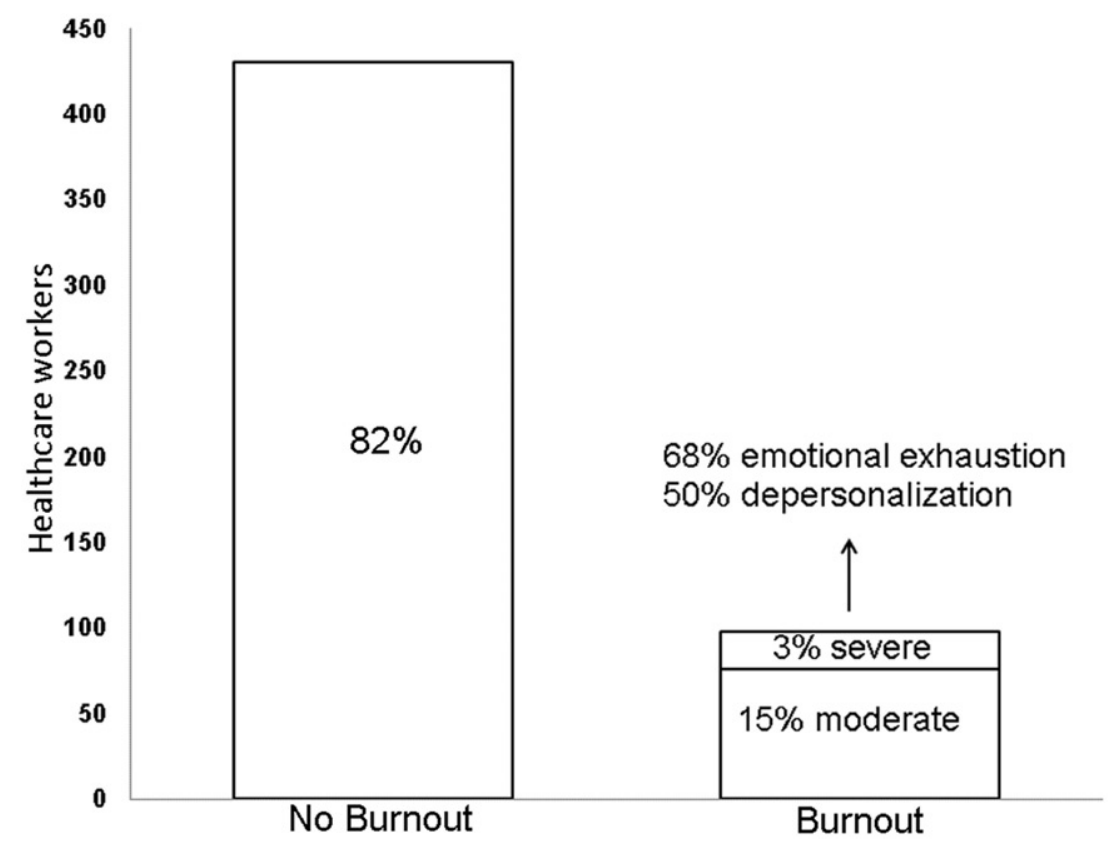

Figure 1. Percentage of burnout among the 523 questionnaires included in the analyses.

Table II. Percentage of burnout and its three sub-components among all participants.

\begin{tabular}{lccr}
\hline & Low & Moderate & High \\
\hline Burnout & $82 \%$ & $15 \%$ & $3 \%$ \\
Emotional exhaustion & $67 \%$ & $21 \%$ & $12 \%$ \\
Depersonalization & $64 \%$ & $27 \%$ & $9 \%$ \\
Accomplishment & $3 \%$ & $12 \%$ & $85 \%$ \\
\hline
\end{tabular}

Table III. Percentage of healthcare workers presenting a burnout.

\begin{tabular}{lr}
\hline Profession & Burnout \\
\hline Physician & $8 \%$ \\
Nurse & $24 \%$ \\
Nursing assistant & $23 \%$ \\
Physio-/speech-/ergo-therapist & $8 \%$ \\
Psychologist/social worker & $10 \%$ \\
\hline
\end{tabular}

Finally, logistic regression showed that the profession was the most associated variable with the burnout (more exactly nurses and nursing assistants) $(z=2.63 ; p=0.009)$.

\section{Discussion}

The aim of this study was to evaluate the prevalence of burnout among healthcare workers taking care of patients with severe disorders of consciousness. The results showed moderate-to-high burnout in $18 \%$ of the participants (defined by severe emotional exhaustion and/or severe depersonalization) suggesting that almost one out of five members of the medical team could potentially suffer from this condition. This is particularly pre-occupying as burnout leads to high rates of absenteeism, lower productivity and represents a significant cost in terms of healthcare [22]. Previous studies using the MBI reported widely varying burnout rates among medical and paramedical teams, ranging from $15-82 \%[10,14,23-26]$. For instance, in several studies, prevalence for burnout in anaesthesia and critical care medicine was present in $\sim 30 \%$ of nurses and $\sim 40-50 \%$ among physicians $[27,28]$. This discrepancy can be explained by the important methodological heterogeneity existing across studies (i.e. recruitment, population, used scale, cut-off score, burnout definition, etc.) [10, 23, 24]. Similar results have been reported in oncology wards [29] where the level of environmental stress is important, as it can be the case when dealing with patients who have suffered severe brain injury and who are unable to communicate.

On the other hand, one third of healthcare workers $(33 \%)$ showed moderate-to-severe emotional exhaustion. This component has been previously related to workload [30] and qualitative job demands in combination with low job resources (e.g. autonomy, social support, supervision relationship and performance feedback) [31]. Moreover, in this study, one third (36\%) of the responders also presented moderate-to-severe depersonalization. These last two results are in line with previous 
Table IV. Chi-square statistics and p-values for the burnout and its three sub-components as regards the working place, profession, age, gender and expertise.

\begin{tabular}{|c|c|c|c|c|c|c|c|c|}
\hline & \multicolumn{2}{|c|}{ Burnout } & \multicolumn{2}{|c|}{ Emotional exhaustion } & \multicolumn{2}{|c|}{ Depersonalization } & \multicolumn{2}{|c|}{ Personal accomplishment } \\
\hline & $\chi^{2}$ & $p$-value & $\chi^{2}$ & $p$-value & $\chi^{2}$ & $p$-value & $\chi^{2}$ & $p$ \\
\hline Working place & 6.6 & $0.01^{\star}$ & 4.3 & 0.11 & 1.16 & 0.56 & 3.8 & 0.15 \\
\hline Profession & 20.1 & $<0.0001^{\star}$ & 20.5 & $<0.009^{\star}$ & 11.9 & 0.15 & 12 & 0.15 \\
\hline Nurse/nursing assistant & 19.9 & $<0.0001^{\star}$ & 18.3 & $<0.0001^{\star}$ & 8.8 & $0.012^{\star}$ & 8.61 & $0.014^{\star}$ \\
\hline Time & 5.7 & $<0.02^{\star}$ & 0.29 & 0.86 & 10.8 & $0.005^{\star}$ & 11.82 & $0.003^{\star}$ \\
\hline Age & 0.5 & 0.48 & 0.28 & 0.87 & 4.89 & 0.09 & 6.22 & $0.04^{\star}$ \\
\hline Gender & 0.94 & 0.33 & 0.53 & 0.77 & 3.64 & 0.16 & 4.6 & 0.1 \\
\hline Expertise & 0.04 & 0.98 & 7.12 & 0.13 & 3.9 & 0.4 & 4.8 & 0.31 \\
\hline
\end{tabular}

${ }^{\star} p<0.05$.

medical studies. Indeed, prevalence of emotional exhaustion and depersonalization among cancer care provider studies seems to be $\sim 36 \%$ and $34 \%$, respectively [29]. Among medical residents, emotional exhaustion has been shown to be $\sim 41-50 \%$ and depersonalization $\sim 34-70 \%$ [23]. Another study including nurses from different specialties reported $23 \%$ of emotional exhaustion and $5 \%$ of depersonalization [5]. Finally, 3\% of this sample presented a low personal accomplishment, with nevertheless an increase of $11 \%$ when assessing burnout participants only. Even though emotional exhaustion and depersonalization rates are higher, low personal accomplishment (and hence low job satisfaction) is important to consider as it can lead clinicians to leave their position. It is particularly relevant as we know that a lack of caregivers decreases quality-of-care and increases job stress which, in its turn, can contribute to burnout [32].

According to the analyses, the variable 'profession' was the most associated with burnout. Nurses and nursing assistants were more likely to suffer from burnout (respectively, 24 and 23\%) than physicians, speech/occupational or physio-therapists, psychologists or social workers (8-10\%). Consistently, the proportion of emotional exhaustion, depersonalization and low personal accomplishment was also greater for those professions in the univariate analysis. Given the nature (e.g. tasks, proximity and time spent with patients) and emotional demands, the nursing team is most likely to develop burnout [5]. The syndrome may be subsequent to a deterioration of relations with the medical team or a difficult relationship between nurses and patients or patients' family. Several studies have also shown that, despite a very positive image of the profession, the majority of nurses in Belgium reported insufficient teamwork, organizational support and social recognition [33]. Moreover, an international study showed that higher levels of burnout among nurses were associated with lower ratings of quality-of-care [34].

Burnout also seemed higher according to the time spent with patients who are unable to communicate. Indeed, the more important the time spent with patients, the greater is the number of healthcare workers with burnout, as previously observed [28]. Moreover, the number of hours worked in a stressful environment may participate in burnout [6]. The proportion of participants with burnout was also more important according to the place of hospitalization and, more particularly, in nursing homes. This result could be explained both by the type of population and by the initial training of the medical staff practicing in a nursing home. Indeed, healthcare workers must deal with chronic patients, often young, who are no longer evolving and whose prognosis is often limited. Because these patients remain in these institutions for a long time, the hope of recovery fades as time goes by and grief may be experienced by caregivers. Additionally, the healthcare team mainly takes care of elderly patients and may feel helpless when faced with management of patients with severe brain damage who need specific care they may not be familiar with. Inadequate preparation [35] and exposure to death [30] can therefore lead to burnout. It is nevertheless difficult to disentangle if burnout is the consequence of working with patients with severe brain injury or more generally the results of working with elderly patients. Further studies should be investigated to answer this question. Meanwhile, recent studies have shown the importance of developing skills in geriatric units in order to prevent emotional exhaustion $[7,36]$. Finally, the number of participants with low personal accomplishment was higher in terms of age, younger caregivers having a higher personal accomplishment than older ones. This difference could potentially be explained by generational differences. Taking the example of the nurses, older caregivers have previously been described as 
idealistic, hard-working and avoiding contexts where they could be confronted to the limit of their knowledge, whereas younger caregivers have been described as realistic, seeking balance between work and family and seeking greater levels of excitement in their work [37]. These differences could lead to low job satisfaction and therefore low personal accomplishment among older caregivers as they may be easily overwhelmed if their life is centred on their work but also because the field of disorders of consciousness has recently increased confronting clinicians with a new expertise to acquire. This constitutes a real problem as the nursing population is getting older [38]. Effective retention strategies (e.g. specific recurrent training, psychological assistance) will have to be developed in order to increase job satisfaction among older caregivers [32].

This study is the first to investigate burnout in caregivers of patients with severe brain injury and disorders of consciousness. Considering the average response rate $(53 \%)$, one should, however, be cautious with the generalization of the findings. One could argue that the percentage of responders among the different professions managing patients recovering from coma was not homogeneous (i.e. more nurses filled in the questionnaires compared to other occupations) which could amplify and/or minimize the effect of burnout within a profession. Nevertheless, the statistical analysis took into account this parameter. Besides, this sample is representative of the composition of a typical medical team as the number of nurses/nursing assistants is usually higher than other professions (such as physicians, speech/occupational or physio-therapists, psychologists or social workers). One could also argue that this study took place in Belgium and could therefore lead to bias based on organizational and governmental issues. Comparison between studies performed in various countries is complicated as findings may differ as a result of differences in cultural, educational and organizational backgrounds [23]. Nevertheless, further investigation is needed to confirm these results and to generalize them across countries and professions.

In conclusion, about one out of five caregivers in this study suffered from burnout. Burnout affects caregivers' wellbeing and therefore the quality-ofcare that may be provided to the patients. Treatment and prevention of burnout are needed to increase the medical staff's quality-of-life and productivity. There are nowadays no preventive or therapeutic measures which could meet the scientific requirements for guidelines [27]. However, several studies highlighted the importance of an effective adjustment (i.e. relaxation, cognitive strategies, communication skills, problem-solving and capacity building of adjustment to stressful situations), a sufficient social support and an appropriate organizational structure to maintain professional satisfaction and good quality-of-care [39, 40]. For example, regular team meetings and supervision sessions may improve the social climate in hospitals and enhance communication [41]. Detecting signs of chronic stress disorder such as work overload and value conflict should be considered as a priority to prevent burnout among medical and paramedical teams working with disorders of consciousness.

\section{Acknowledgements}

We thank all participating centres leaders involved in the Belgian federal project for the care of patients with disorders of consciousness. We also thank Dounia Tchekouteff.

Declaration of Interest: The authors report no conflicts of interest. This study was supported by the Belgian Federal public health, the National Funds for Scientific Research (FNRS), James S. McDonnell Foundation, Mind Science Foundation, European Commission (Mindbridge, DISCOS, DECODER \& COST), Concerted Research Action (ARC 06/11-340), Public Utility Foundation 'Université Européenne du Travail', 'Fondazione Europea di Ricerca Biomedica' and the University of Liège.

\section{References}

1. The Multi-Society Task Force on PVS. Medical aspects of the persistent vegetative state (1). New England Journal of Medicine 1994;330:1499-1508.

2. Laureys S, Celesia G, Cohadon F, Lavrijsen J, León-Carrión J, Sannita WG, Sazbon L, Schmutzhard E, von Wild KR, Zeman A, et al. European Task Force on Disorders of Consciousness. Unresponsive wakefulness syndrome: A new name for the vegetative state or apallic syndrome. BMC Medicine 2010;8:68.

3. Giacino JT, Ashwal S, Childs N, Cranford R, Jennett B, Katz DI, Kelly JP, Rosenberg JH, Whyte J, Zafonte RD, et al. The minimally conscious state: Definition and diagnostic criteria. Neurology 2002;58:349-353.

4. Maslach C, Schaufeli W, Leiter M. Job burnout. Annual Review of Psychology 2001;52:397-422.

5. Sahraian A, Fazelzadeh A, Mehdizadeh A, Toobaee S. Burnout in hospital nurses: A comparison of internal, surgery, psychiatry and burns wards. International Nursing Review 2008;55:62-7.

6. Chen S, McMurray A. "Burnout" in intensive care nurses. Journal of Nursing Research 2001;9:152-164.

7. Cocco E, Gatti M, de Mendonca Lima C, Camus V. A comparative study of stress and burnout among staff caregivers in nursing homes and acute geriatric wards. International Journal of Geriatric Psychiatry 2003;18: 78-85. 
8. Trufelli D, Bensi C, Garcia J, Narahara JL, Abrão $M N$, Diniz RW, Miranda Vda C, Soares H. P, Del Giglio A. Burnout in cancer professionals: A systematic review and meta-analysis. European Journal of Cancer Care 2008;17: $524-531$

9. Książek I, Stefaniak T, Stadnyk M, Książek J. Burnout syndrome in surgical oncology and general surgery nurses: A cross-sectional study. European Journal of Oncology Nursing 2011;15:347-350.

10. Hyman S, Michaels D, Berry J, Schildcrout J, Mercaldo N, Weinger M. Risk of burnout in perioperative clinicians: A survey study and literature review. Anesthesiology 2011;114: 194-204.

11. Gundersen L. Physician burnout. Annals of Internal Medicine 2001;135:145-148.

12. Shanafelt T, Bradley K, Wipf J, Back A. Burnout and selfreported patient care in an internal medicine residency program. Annals of Internal Medicine 2002;136:358-367.

13. Abrahamson K, Jill Suitor J, Pillemer K. Conflict between nursing home staff and residents' families: Does it increase burnout? Journal of Aging and Health 2009;21:895-912.

14. Wallace J, Lemaire J, Ghali W. Physician wellness: A missing quality indicator. Lancet 2009;14:1714-1721.

15. McHugh M, Kutney-Lee A, Cimiotti J, Sloane D, Aiken L. Nurses' widespread job dissatisfaction, burnout, and frustration with health benefits signal problems for patient care. Health Affairs (Millwood) 2011;30:202-210.

16. McLaughlin A, Erdman J. Rehabilitation staff stress as it relates to patient acuity and diagnosis. Brain Injury 1992;6:59-64.

17. Duckett S. Staff stress in head injury rehabilitation. Brain Injury 1996;10:133-138.

18. Chiambretto P, Rossi Ferrario S, Zotti A. Patients in a persistent vegetative state: Caregiver attitudes and reactions. Acta Neurologica Scandinavica 2001;104:364-368.

19. Schuttle N, Toppinen S, Kalimo R, Schaufeli W. The factorial validity of the Maslach Burnout Inventory-General Survey (MBI-GS) across occupational groups and nations. Journal of Occupational and Organizational Psychology 2000;73:53-66.

20. Kilfedder CJ, Power KG, Wells TJ. Burnout in psychiatric nursing. Journal of Advanced Nursing 2001;34:383-396.

21. Maslach C, Jackson SE. Maslach Burnout Inventory. Palo Alto, CA: Consulting Psychologist Press; 1981.

22. Hallsten L, Voss M, Stark S, Josephson M. Job burnout and job wornout as risk factors for long-term sickness absence. Work 2011;38:181-192.

23. Prins J, Gazendam-Donofrio S, Tubben B, van der Heijden F, van de Wiel H, Hoekstra-Weebers J. Burnout in medical residents: A review. Medical Education 2007;41: 788-800.

24. Paris MJ, Hoge M. Burnout in the mental health workforce: A review. Journal of Behavioral Health Services \& Research 2010;37:519-528.

25. Arigoni F, Bovier P, Sappino A. Trend of burnout among Swiss doctors. Swiss Medical Weekly 2010;140:w13070.
26. Embriaco N, Azoulay E, Barrau K, Kentish N, Pochard F, Loundou A, Papazian L. High level of burnout in intensivists: Prevalence and associated factors. American Journal of Respiratory and Critical Care Medicine 2007;175:686-692.

27. Michalsen A, Hillert A. Burnout in anesthesia and intensive care medicine. Part 2: Epidemiology and importance for the quality of care. Anaesthesist 2011;60:31-38.

28. Embriaco N, Papazian L, Kentish-Barnes N, Pochard F, Azoulay E. Burnout syndrome among critical care healthcare workers. Current Opinion in Critical Care 2007;13:482-488.

29. Trufelli D, Bensi C, Garcia J, Narahara JL, Abrão $M N$, Diniz RW, Miranda Vda C, Soares HP, Del Giglio A. Burnout in cancer professionals: A systematic review and meta-analysis. European Journal of Cancer Care 2008;17: 524-531.

30. Greenglass E, Burke R, Fiksenbaum L. Workload and burnout in nurses. Journal of Community \& Applied Social Psychology 2001;11:211-215.

31. Bakker A, Demerouti E, Eeuwema M. Job resources buffer the impact of job demands on burnout. Journal of Occupational Psychology 2005;10:170-180.

32. Fitzgerald DC. Aging, experienced nurses: Their value and needs. Contemporary Nurse 2007;24:237-242.

33. Siebens K, Casterle B, Abraham I, Dierckx K, Braes T, Darras E, Dubois Y, Milisen K, representing the BELIMAGE group. The professional self-image of nurses in Belgian hospitals: A cross-sectional questionnaire survey. International Journal of Nursing Studies 2006;43:71-82.

34. Poghosyan L, Clarke S, Finlayson M, Aiken L. Nurse burnout and quality of care: Cross-national investigation in six countries. Research in Nursing \& Health 2010;33: 288-298.

35. Cameron C, Pirozzo S, Tooth L. Long-term care of people below age 65 with severe acquired brain injury: Appropriateness of aged care facilities. Australian and New Zealand Journal of Public Health 2001;25:261-264.

36. Hasson H, Arnetz J. The impact of an educational intervention on nursing staff ratings of quality of older people care: A prospective, controlled intervention study. International Journal of Nursing Studies 2009;46:470-478.

37. Keepnews D, Brewer C, Kovner C, Shin J. Generational differences among newly licensed registered nurses. Nursing Outlook 2010;58:155-163.

38. Norman L, Donelan K, Buerhaus P, Willis G, Williams M, Ulrich B, Dittus R. The older nurse in the workplace: Does age matter? Nursing Economics 2005;23:282-289, 279.

39. Piko B. Burnout, role conflict, job satisfaction and psychosocial health among Hungarian health care staff: A questionnaire survey. International Journal of Nursing Studies 2006;43:311-318.

40. Braithwaite M. Nurse burnout and stress in the NICU. Advances in Neonatal Care 2008;8:343-347.

41. Kowalski C, Ommen O, Driller E, Ernstmann N, Wirtz MA, Köhler T, Pfaff H. Burnout in nurses-the relationship between social capital in hospitals and emotional exhaustion. Journal of Clinical Nursing 2010;19:1654-1663. 